\title{
Rare-Variant Kernel Machine Test for Longitudinal Data from Population and Family Samples
}

\author{
Qi Yan ${ }^{a}$ Daniel E. Weeks ${ }^{b}$ Hemant K. Tiwaric Nengjun $\mathrm{Yi}^{\mathrm{c}}$ Kui Zhang $^{\mathrm{d}}$ \\ Guimin Gao ${ }^{e}$ Wan-Yu Ling ${ }^{g}$ Xiang-Yang Lou ${ }^{f}$ Wei Chen ${ }^{a, b}$ Nianjun Liu $^{c}$ \\ aDivision of Pulmonary Medicine, Allergy and Immunology, Department of Pediatrics, Children's Hospital of \\ Pittsburgh of UPMC, and bepartments of Human Genetics and Biostatistics, University of Pittsburgh, \\ Pittsburgh, Pa., 'Department of Biostatistics, University of Alabama at Birmingham, Birmingham, Ala., \\ ${ }^{\mathrm{d}}$ Department of Mathematical Sciences, Michigan Technological University, Houghton, Mich., e Department of \\ Public Health, University of Chicago, Chicago, III., and fDepartment of Biostatistics and Bioinformatics, \\ Tulane University, New Orleans, La., USA; IInstitute of Epidemiology and Preventive Medicine, College of \\ Public Health, National Taiwan University, Taipei, Taiwan, ROC
}

\section{Key Words}

Rare variant $\cdot$ Longitudinal data $\cdot$ Linear mixed model · Linear kernel function · Family samples

\begin{abstract}
Objective: The kernel machine (KM) test reportedly performs well in the set-based association test of rare variants. Many studies have been conducted to measure phenotypes at multiple time points, but the standard KM methodology has only been available for phenotypes at a single time point. In addition, family-based designs have been widely used in genetic association studies; therefore, the data analysis method used must appropriately handle familial relatedness. A rare-variant test does not currently exist for longitudinal data from family samples. Therefore, in this paper, we aim to introduce an association test for rare variants, which includes multiple longitudinal phenotype measurements for either population or family samples. Methods: This approach uses KM regression based on the linear mixed model framework and is applicable to longitudinal data from either
\end{abstract}

\section{KARGER 125}

() 2016 S. Karger AG, Base

0001-5652/16/0803-0126\$39.50/0

E-Mail karger@karger.com

www.karger.com/hhe population (L-KM) or family samples (LF-KM). Results: In our population-based simulation studies, L-KM has good control of Type I error rate and increased power in all the scenarios we considered compared with other competing methods. Conversely, in the family-based simulation studies, we found an inflated Type I error rate when L-KM was applied directly to the family samples, whereas LF-KM retained the desired Type I error rate and had the best power performance overall. Finally, we illustrate the utility of our proposed LF-KM approach by analyzing data from an association study between rare variants and blood pressure from the Genetic Analysis Workshop 18 (GAW18). Conclusion: We propose a method for rare-variant association testing in population and family samples using phenotypes measured at multiple time points for each subject. The proposed method has the best power performance compared to competing approaches in our simulation study.

C 2016 S. Karger AG, Basel
Nianjun Liu

RPHB 420A

1665 University Blvd.

Birmingham, AL 35294 (USA)

E-Mail nliu@uab.edu

Wei Chen

Rangos Research Building, 9th Floor

4401 Penn Ave.

Pittsburgh, PA 15224 (USA)

E-Mail wei.chen@chp.edu 


\section{Introduction}

Genome-wide association studies (GWAS) have been widely used to identify common genetic variants that are associated with complex human diseases [1-5]. For a typical GWAS, hundreds or thousands of subjects are recruited and genotyped at hundreds of thousands of genetic variants [e.g., single nucleotide polymorphisms (SNPs)]. An association between the phenotype and each of the SNPs is usually tested sequentially via a singlemarker test. However, the traditional single-marker association test is not powerful enough to detect rare variants that may play key roles in influencing complex diseases $[6,7]$. To increase the statistical power, many set-based methods [7-17] have been developed to evaluate the joint effects of a group of genetic variants in a predefined genomic region on the phenotypes of interest. Among the existing methods, one powerful, flexible, and computationally efficient test is the sequence kernel machine-based association test (SKAT) $[18,19]$. In this kernel machine (KM) approach, weights are assigned to each marker to improve the power. In addition, KM can easily include other covariates in the model. Both linear and nonlinear kernels may be used for the genotype-phenotype relationship. Furthermore, the KM test statistic follows a mixture of $\chi^{2}$ distributions. Thus, $p$ values can be computed analytically and quickly without employing resampling.

In many genetic studies, phenotypes are measured at multiple time points for each subject [20-23]. Quite a few methods [24-27] have been developed for longitudinal genetic data analysis, but very few of these methods are for rare variants [24]. We believe that a method in which all time points are accounted for jointly in an association test would likely improve the power. In addition, family-based designs have been widely used to study the association between diseases and genetic factors [28-33]. In GWAS that employ population samples, the association between quantitative phenotypes and genetic markers is usually investigated by applying a general linear model; however, statistics based on general linear models result in inflated Type I error rates when applied to family data directly [34-36] because they ignore familial correlations. Instead of a general linear model, a linear mixed model including a random polygenic effect can be used to handle the familial correlation. The covariance of random polygenic effects in all subjects can be expressed by a kinship matrix. This linear mixed model with a kinship matrix has typically been used when dealing with family samples in GWAS
[37]. SKAT was recently extended to family samples by including the kinship matrix [38-42].

In this study, we propose a method for rare-variant association testing in population and family samples using phenotypes measured at multiple time points for each subject. In our population-based simulation studies, we found that when the longitudinal kernel machine (L-KM) test considered all time points, it was more powerful than approaches that only considered some of the time points, although all the methods we tested had correct Type I error rates. Conversely, in our family-based simulation studies, we found an inflated Type I error rate when L-KM was directly applied to the family sample, whereas the longitudinal family kernel machine (LF-KM) test retained the desired Type I error rate and had the best power overall compared with the methods that only considered some of the time points. Another study reported similar findings [24]. Finally, we assessed the association between rare genetic variants and blood pressure from the Genetic Analysis Workshop 18 (GAW18) data to demonstrate our approach. These data present challenges for analysis, including large pedigrees, repeated measures, and whole-genome sequence data [43-45].

\section{Methods}

KM Regression in a Linear Mixed Model Framework

We set the stage for our subsequent derivations by defining, for a trait measured at a single time point, a linear mixed model setup similar to that of Chen et al. [38]. Let there be $n$ subjects with $q$ genetic variants. The $n \times 1$ vector of the quantitative trait $y$ follows a linear mixed model:

$$
\mathbf{y}=\mathbf{X} \boldsymbol{\beta}+\mathbf{G} \boldsymbol{\gamma}+\mathbf{u}+\boldsymbol{\varepsilon},
$$

where $\mathbf{X}$ is an $n \times p$ covariate matrix, $\boldsymbol{\beta}$ is a $p \times 1$ vector containing parameters for the fixed effects (an intercept and $p-1$ covariates), $\mathbf{G}$ is an $n \times q$ genotype matrix for the $q$ genetic variants of interest where an additive genetic model is assumed (i.e., coded as 0,1 , or 2 representing the copies of minor alleles) for illustration, $\gamma$ is a $q \times 1$ vector for the random effects of the $q$ genetic variants, $\mathbf{u}$ is an $n \times 1$ vector for the random effects due to covariates (e.g., time for longitudinal data or relatedness in families), and $\varepsilon$ is an $n \times 1$ vector for the random error. The random effect $\gamma_{j}$ for variant $j$ is assumed to be normally distributed with mean zero and variance $\tau w_{j}$; thus, the null hypothesis $H_{0}: \gamma=0$ is equivalent to $H_{0}: \tau=0$, which can be tested with a variance component score test [19] in the mixed model. The random variables $\boldsymbol{\varepsilon}$ and $\mathbf{u}$ are also assumed to be normally distributed, and are uncorrelated with each other and also with $\gamma$ :

$$
\begin{aligned}
& \boldsymbol{\gamma} \sim N(0, \tau \mathbf{W}) \\
& \mathbf{u} \sim N(0, \mathbf{K}) \\
& \boldsymbol{\varepsilon} \sim N\left(0, \sigma_{\mathrm{E}}^{2} \mathbf{I}\right),
\end{aligned}
$$


where $\mathbf{W}$ is a predefined $q \times q$ diagonal weight matrix for each variant and may use

$$
\sqrt{w_{i}}=\operatorname{Beta}\left(M A F_{i}, 1,25\right)
$$

as in SKAT, $\mathbf{K}$ is an $n \times n$ covariance matrix, and $\sigma_{E}^{2}$ is the error variance.

Following the same rationale as in the derivation of the SKAT score statistic [46-48] (refer to the Appendix for a detailed derivation), the test statistic is:

$$
\mathrm{Q}=(\mathbf{y}-\mathbf{X} \hat{\boldsymbol{\beta}})^{\prime} \hat{\Sigma}^{-1} \mathbf{G W G}^{\prime} \hat{\Sigma}^{-1}(\mathbf{y}-\mathbf{X} \hat{\boldsymbol{\beta}}),
$$

where $\hat{\boldsymbol{\beta}}$ is the vector of estimated fixed effects of covariates under $H_{0}$, and $\hat{\Sigma}=\hat{\mathbf{K}}+\hat{\sigma}_{E}^{2} \mathbf{I}$ is the estimated variance-covariance matrix under $H_{0}$. The statistic $Q$ is a quadratic form of $(\mathbf{y}-\mathbf{X} \hat{\boldsymbol{\beta}})$, and therefore follows a mixture of $\chi^{2}$ distributions [49], although some of the parameters are estimated [50]. Thus, the $p$ values can be calculated by numerical algorithms, such as moment-matching methods [47, 51], Davies' method [52], and Kuonen's saddlepoint method [53]. In this study, we used the Davies method.

\section{L-KM Regression for Quantitative Traits in Population Data}

We now extend the above model by incorporating intercept and time as both fixed and random effects. Different covariance structures for longitudinal data, such as compound symmetry, autoregressive, and Toeplitz, can be easily implemented under this framework.

Under the null hypothesis, $H_{0}: \tau=0$, for the $i$-th subject at time point $j$, the random intercept and time model is:

$$
y_{i j}=\beta_{0}+t_{i j} \beta_{1}+b_{0 i}+t_{i j} b_{1 i}+\varepsilon_{i j}
$$

where $t_{i j}$ indicates time, $\beta_{0}$ and $\beta_{1}$ are the fixed effects of intercept and time, while $b_{0 i}$ and $b_{1 i}$ are the random effects of intercept and time for the $i$-th subject. For one subject, the model can be rewritten as

$$
\mathbf{y}_{i}=\mathbf{X}_{i} \boldsymbol{\beta}+\mathbf{Z}_{i} \mathbf{b}_{i}+\boldsymbol{\varepsilon}_{i}
$$

We assume that there are $m$ time points. Thus, $\mathbf{y}_{i}=\left(y_{i 1}, y_{i 2}, \ldots, y_{i m}\right)^{\prime}$ is an $m \times 1$ vector, $\mathbf{X}_{i}$ is an $m \times 2$ matrix for intercept and time, $\boldsymbol{\beta}=\left(\begin{array}{ll}\beta_{0} & \beta_{1}\end{array}\right)$ and $\mathbf{b}_{i}=\left(\begin{array}{lll}b_{0 i} & b_{1 i}\end{array}\right)$. For simplicity, we did not include other covariates (which can be easily included) in the model; therefore, $\mathbf{Z}_{i}$ is the same as $\mathbf{X}_{i}$, and

$$
\begin{aligned}
& \operatorname{Var}\left(\mathbf{b}_{i}\right)=\left(\begin{array}{ll}
\sigma_{i n t}^{2} & \sigma_{c o v} \\
\sigma_{c o v} & \sigma_{\text {time }}^{2}
\end{array}\right) \\
& \operatorname{Var}\left(\mathbf{y}_{i}\right)=\mathbf{Z}_{i} \operatorname{Var}\left(\mathbf{b}_{i}\right) \mathbf{Z}_{i}{ }^{\prime}+\sigma_{E}^{2} \mathbf{I}_{m \times m} .
\end{aligned}
$$

For the whole dataset, the variance term is:

$$
\operatorname{Var}(\mathbf{y})=\mathbf{I} \otimes \mathbf{Z}_{i} \operatorname{Var}\left(\mathbf{b}_{i}\right) \mathbf{Z}_{i}^{\prime}+\sigma_{E}^{2} \mathbf{I}=\mathbf{\Sigma},
$$

where $\mathbf{y}$ is an $n \times m \times 1$ vector, and $\otimes$ is the Kronecker product to produce a diagonal block matrix. The variance terms $\sigma_{\text {int }}^{2}, \sigma_{\text {time }}^{2}, \sigma_{c o v}$, and $\sigma_{E}^{2}$ can be estimated from the data (e.g., using the R package nlme [54]), and then the L-KM test statistic Q can be constructed in the same way as in the above section.
LF-KM Regression for Quantitative Traits of Family Data

For pedigree data, familial correlation can be added to the model as an additional random variable. Under the null hypothesis, $H_{0}: \tau=0$, for the $i$-th subject in the $k$-th family at time point $j$, the random intercept and time model becomes:

$$
y_{i j k}=\beta_{0}+t_{i j k} \beta_{1}+b_{0 i k}+t_{i j k} b_{1 i k}+\delta_{i k}+\varepsilon_{i j k},
$$

where $\beta_{0}$ and $\beta_{1}$ are the fixed effects of intercept and time, while $b_{0 i k}$ and $b_{1 i k}$ are the random effects of intercept and time. $\delta_{i k}$ is the random effect for familial correlation. For one subject with $m$ time point observations, the model can be rewritten in vector form as:

$$
\mathbf{y}_{i k}=\mathbf{X}_{i k} \boldsymbol{\beta}+\mathbf{Z}_{i k} \mathbf{b}_{i k}+\delta_{i k}+\boldsymbol{\varepsilon}_{i k} .
$$

Again, we assume $m$ time points and no other covariates; thus, $\mathbf{y}_{i k}$ is an $m \times 1$ vector, $\mathbf{X}_{i k}$ and $\mathbf{Z}_{i k}$ are the same $m \times 2$ matrix for intercept and time. For illustration, we consider the model for a trio family:

$$
\begin{aligned}
& \mathbf{y}_{k}=\mathbf{X}_{k} \boldsymbol{\beta}+\mathbf{Z}_{k} \mathbf{b}_{k}+\boldsymbol{\delta}_{k}+\boldsymbol{\varepsilon}_{k} \\
& \operatorname{Var}\left(\mathbf{Z}_{k} \mathbf{b}_{k}\right)=\mathbf{I}_{3 \times 3} \otimes \mathbf{Z}_{i k} \operatorname{Var}\left(\mathbf{b}_{i k}\right) \mathbf{Z}_{i k}{ }^{\prime}=\mathbf{I}_{3 \times 3} \otimes \mathbf{Z}_{i k}\left(\begin{array}{ll}
\sigma_{\text {int }}^{2} & \sigma_{c o v} \\
\sigma_{\text {cov }} & \sigma_{\text {time }}{ }^{2}
\end{array}\right) \mathbf{Z}_{i k}{ }^{\prime} \\
& \operatorname{Var}\left(\boldsymbol{\delta}_{k}\right)=\sigma_{G}^{2} \times \mathbf{J}_{k} \boldsymbol{\Phi}_{k} \mathbf{J}_{k}{ }^{\prime} \\
& =\sigma_{G}^{2} \times\left[\begin{array}{ccc}
1_{m \times 1} & 0_{m \times 1} & 0_{m \times 1} \\
0_{m \times 1} & 1_{m \times 1} & 0_{m \times 1} \\
0_{m \times 1} & 0_{m \times 1} & 1_{m \times 1}
\end{array}\right] \boldsymbol{\Phi}_{k}\left[\begin{array}{ccc}
1_{m \times 1} & 0_{m \times 1} & 0_{m \times 1} \\
0_{m \times 1} & 1_{m \times 1} & 0_{m \times 1} \\
0_{m \times 1} & 0_{m \times 1} & 1_{m \times 1}
\end{array}\right]^{\prime} \\
& \Phi_{k}=\left[\begin{array}{ccc}
1 & 0 & 0.5 \\
0 & 1 & 0.5 \\
0.5 & 0.5 & 1
\end{array}\right]
\end{aligned}
$$

where $\mathbf{y}_{k}$ is a $3 m \times 1$ vector, and $\boldsymbol{\Phi}_{k}$ is twice the kinship matrix for a trio family:

$$
\operatorname{Var}\left(\mathbf{y}_{k}\right)=\operatorname{Var}\left(\mathbf{Z}_{k} \mathbf{b}_{k}\right)+\operatorname{Var}\left(\boldsymbol{\delta}_{k}\right)+\sigma_{E}^{2} \mathbf{I}_{3 m \times 3 m}
$$

For the whole dataset with multiple families, we assume $n$ individuals from the families. The variance term is:

$$
\begin{aligned}
\operatorname{Var}(\mathbf{y}) & =\mathbf{I} \otimes \mathbf{Z}_{i k} \operatorname{Var}\left(\mathbf{b}_{i k}\right) \mathbf{Z}_{i k}{ }^{\prime}+\sigma_{G}^{2} \times \mathbf{J} \Phi \mathbf{J}^{\prime}+\sigma_{E}^{2} \mathbf{I}=\sum \\
\mathbf{J} & =\left[\begin{array}{ccc}
1_{m \times 1} & \ldots & 0_{m \times 1} \\
\vdots & \ddots & \vdots \\
0_{m \times 1} & \cdots & 1_{m \times 1}
\end{array}\right]_{n m \times n},
\end{aligned}
$$

where $\sigma_{\text {int }}^{2}, \sigma_{\text {time }}^{2}, \sigma_{\text {cov }}$, and $\sigma_{E}^{2}$ represent the same variance/covariance terms as in the population-based model. $\sigma_{G}^{2}$ represents the variance term for the random effects of familial correlation. $\Phi$ is twice the $n \times n$ kinship matrix obtained from the data. All the variance terms can be estimated (e.g., using the R package pedigreemm [55]), and then the LF-KM test statistic Q can be constructed as above. 


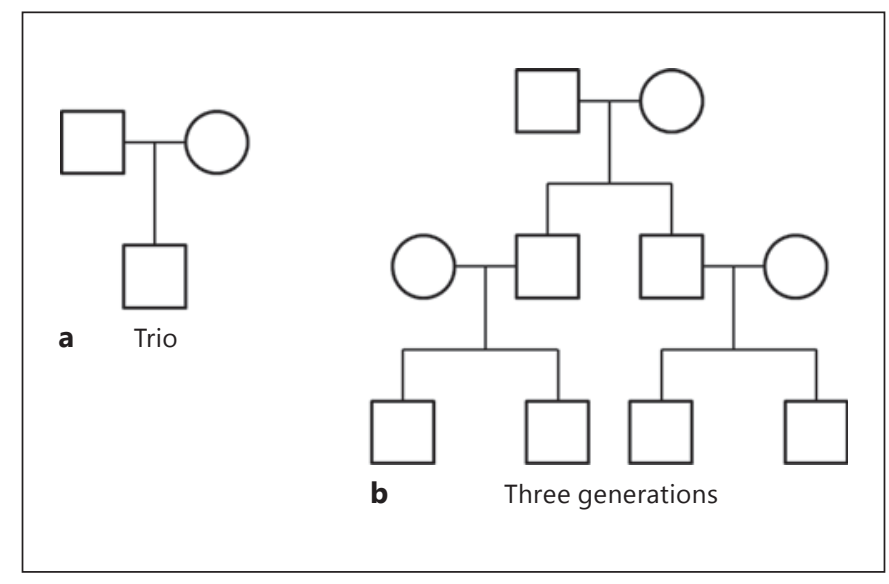

Fig. 1. Two pedigree structures used in the simulation studies.

\section{Population-Based Simulation Study}

Simulation of Sample Genotypes

We simulated 1,000 unrelated samples based on a matrix of 10,000 haplotypes over a 200 -kb region generated by the calibrated coalescent model [56], mimicking the European ancestry linkage disequilibrium (LD) structure. Only rare variants [minor allele frequency (MAF) <0.05] were kept, and 2,000 haplotypes were randomly selected to form the unrelated subjects' haplotypes. Then, 30 neighboring SNPs with at least one copy of the minor allele (i.e., excluding non-polymorphic variants) were used in the analysis. We simulated 100 genotype datasets.

Type I Error Rate

For each of the 100-genotype datasets generated, we simulated 1,000 sets of quantitative longitudinal phenotypes based on five time points. The phenotypes for subject $i$ were generated via the model:

$$
\mathbf{y}_{i}=0.05 \times \mathbf{X}_{1 i}+0.5 \times \mathbf{X}_{2 i}+0.1 \times \mathbf{t}_{i}+\mathbf{v}_{i},
$$

where $\mathbf{X}_{1 i}$ is a continuous covariate generated from a normal distribution with a mean of 50 and a standard deviation of 5 (this single value was repeated five times to mimic the five time points); $\mathbf{X}_{2 i}$ is a dichotomous covariate generated from a Bernoulli distribution with a probability of 0.5 , which was also repeated five times; $\mathbf{t}_{i}$ is the time point, assuming values of $0,1,2,3$, or 4 , and $\mathbf{v}_{i}$ is random error that follows a multivariate normal distribution with a mean of 0 , and covariance matrix $\operatorname{Var}\left(\mathbf{y}_{i}\right)$.

$$
\begin{aligned}
\operatorname{Var}\left(\mathbf{y}_{i}\right) & =\mathbf{Z}_{i}\left(\begin{array}{ll}
\sigma_{\text {int }}^{2} & \sigma_{\text {cov }} \\
\sigma_{\text {cov }} & \sigma_{\text {time }}^{2}
\end{array}\right) \mathbf{Z}_{i}{ }^{\prime}+\sigma_{E}^{2} \mathbf{I}_{5 \times 5} \\
& =\left(\begin{array}{ll}
1 & 0 \\
1 & 1 \\
1 & 2 \\
1 & 3 \\
1 & 4
\end{array}\right)\left(\begin{array}{cc}
1 & -0.5 \\
-0.5 & 1
\end{array}\right)\left(\begin{array}{ccccc}
1 & 1 & 1 & 1 & 1 \\
0 & 1 & 2 & 3 & 4
\end{array}\right)+\mathbf{I}_{5 \times 5},
\end{aligned}
$$

where the variance of random effects of intercept and time as well as random error were set to 1 , the covariance between the intercept and time was set to -0.5 , which assumes the rate of change is slow when the baseline value is large (e.g., it is less likely that a patient with high blood pressure at baseline will quickly reach an even higher blood pressure). The phenotypes of all of the individuals were generated in the same manner, and the 1,000 sets of simulated phenotypes for each of the 100-genotype datasets were used to evaluate the Type I error rate.

Using these unrelated population samples, we compared L-KM using all five time points (abbreviated as L-KM-m5) with four other approaches: (1) L-KM using a subset of three time points (LKM-m3), (2) L-KM with $20 \%$ of the observations randomly assigned to missing (L-KM-missing), (3) KM on the averaged phenotype over five time points (avg-KM), and (4) KM on the phenotype at the last time point (last-KM).

\section{Power Evaluation}

We generated the same genotypes as described above. The quantitative phenotypes for one subject were generated via the following model:

$$
\mathbf{y}_{i}=0.05 \times \mathbf{X}_{1 i}+0.5 \times \mathbf{X}_{2 i}+0.1 \times \mathbf{t}_{i}+\sum_{j=1}^{q} \beta_{j} \mathbf{G}_{j}+\mathbf{v}_{i},
$$

where $\mathbf{X}_{1}, \mathbf{X}_{2 i}, \mathbf{t}_{i}$, and $\mathbf{v}_{i}$ were set up the same way as described above. $\mathbf{G}_{1}, \mathbf{G}_{2}, \ldots, \mathbf{G} q$ are the genotypes of causal SNPs, and $\beta_{1}$, $\beta_{2}, \ldots, \beta_{q}$ are coefficients of the causal SNPs. We assumed that $30 \%$ of all variants are disease susceptibility variants. Furthermore, each $\beta_{j}$ was set as $c\left|\log _{10} M A F_{j}\right|$ in order to assign large weights to rare variants, where $c=0.4$ was chosen, such that when $\mathrm{MAF}=0.0001$, $\beta=1.6$, following the literature [19]. Because causal variants might not influence the phenotype in a consistent direction, we also assumed that one third of the causal variants were protective (i.e., $20 \%$ risk variants and $10 \%$ protective variants), with $\beta_{j}=-c \mid \log _{10}$ $M A F_{j} \mid$. The phenotypes for all of the individuals were generated in the same manner, and the 1,000 sets of simulated phenotypes for each of the 100-genotype datasets were used to evaluate power. We compared L-KM-m5 with L-KM-m3, L-KM-missing, avg$\mathrm{KM}$, and last-KM.

\section{Family-Based Simulation Study}

Simulation of Sample Genotypes

To simulate family data, we used the aforementioned pool of 10,000 haplotypes over a $200-\mathrm{kb}$ region and family structures as shown in figure 1 . First, haplotypes were randomly selected for all founders. The offspring haplotypes were generated by randomly transmitting one of the two haplotypes of each parent to the child. For the scenario of trio families (fig. 1a), we generated 300 families with a father, a mother, and one offspring in each family. For the three-generation scenario, we generated 100 families (fig. 1b). Furthermore, 30 neighboring polymorphic SNPs were used in the analysis, and we simulated 100-genotype datasets for each of the two scenarios.

\section{Type I Error Rate}

To evaluate the Type I error rates, we simulated 1,000 sets of phenotypes for each of the 100-genotype datasets. The quantitative phenotypes for one trio family were generated via the following model:

$$
\mathbf{y}_{k}=0.05 \times \mathbf{X}_{1 k}+0.5 \times \mathbf{X}_{2 k}+0.1 \times \mathbf{t}_{k}+\boldsymbol{\delta}_{k}+\mathbf{v}_{k},
$$

where $\mathbf{X}_{1 k}$ and $\mathbf{X}_{2 k}$ are the same as described above. Both $\mathbf{X}_{1 k}$ and $\mathbf{X}_{2 k}$ repeat five times for five time points for one subject, and each 
family includes three subjects; time $\mathbf{t}_{k}$ is a sequence of $0,1,2,3,4$, $0,1,2,3,4,0,1,2,3,4 ; \mathbf{v}_{k}$ follows a multivariate normal distribution with means of 0 and a covariance matrix, $\operatorname{Var}\left(\mathbf{y}_{k}\right)$ :

$$
\begin{aligned}
& \operatorname{Var}\left(\mathbf{y}_{k}\right)=\mathbf{I}_{3 \times 3} \otimes \mathbf{Z}_{i k}\left(\begin{array}{ll}
\sigma_{i n t}^{2} & \sigma_{c o v} \\
\sigma_{c o v} & \sigma_{\text {time }}^{2}
\end{array}\right) \mathbf{Z}_{i k}{ }^{\prime}+\sigma_{G}^{2} \times \mathbf{J}_{k} \Phi_{k} \mathbf{J}_{k}{ }^{\prime}+\sigma_{E}^{2} \mathbf{I}_{15 \times 15}= \\
& \mathbf{I}_{3 \times 3} \otimes\left(\begin{array}{ll}
1 & 0 \\
1 & 1 \\
1 & 2 \\
1 & 3 \\
1 & 4
\end{array}\right)\left(\begin{array}{cc}
1 & -0.5 \\
-0.5 & 1
\end{array}\right)\left(\begin{array}{ccccc}
1 & 1 & 1 & 1 & 1 \\
0 & 1 & 2 & 3 & 4
\end{array}\right)+ \\
& {\left[\begin{array}{lll}
1_{5 \times 1} & 0_{5 \times 1} & 0_{5 \times 1} \\
0_{5 \times 1} & 1_{5 \times 1} & 0_{5 \times 1} \\
0_{5 \times 1} & 0_{5 \times 1} & 1_{5 \times 1}
\end{array}\right]\left[\begin{array}{ccc}
1 & 0 & 0.5 \\
0 & 1 & 0.5 \\
0.5 & 0.5 & 1
\end{array}\right]\left[\begin{array}{ccc}
1_{5 \times 1} & 0_{5 \times 1} & 0_{5 \times 1} \\
0_{5 \times 1} & 1_{5 \times 1} & 0_{5 \times 1} \\
0_{5 \times 1} & 0_{5 \times 1} & 1_{5 \times 1}
\end{array}\right]^{\prime}+\mathbf{I}_{15 \times 15},}
\end{aligned}
$$

where the variance of the random effects of intercept, time, genetic variance, and error were set to 1 , and the covariance between the intercept and time was set to -0.5 . The three-generation family scenario was set up in an analogous way, but with a more complicated kinship matrix. In this work, the kinship coefficients were directly obtained from the known pedigree structures. If genome-wide genotype data are available, it may be more advantageous to use genetic markers to estimate the kinship coefficients [57-62]. Actually, with enough genetic markers to estimate kinship coefficients, the proposed method is a unified approach that allows for any relationship in the data, even cryptic relatedness.

We compared the Type I error rates of LF-KM using all five time points (abbreviated as LF-KM-m5) to the Type I error rates of five other approaches: (1) LF-KM using a subset of three time points (LF-KM-m3), (2) LF-KM with $20 \%$ of the observations randomly assigned to missing (LF-KM-missing), (3) family KM on the averaged phenotype over five time points (avg-F-KM), (4) family $\mathrm{KM}$ on the last time point phenotype (last-F-KM), and (5) L-KM-m5.

\section{Power Evaluation}

For the causal gene sets, we used the same genotypes as described in the evaluation of the Type I error rates. The quantitative phenotypes for one family were generated via the model:

$$
\mathbf{y}_{k}=0.05 \mathbf{X}_{1 k}+0.5 \mathbf{X}_{2 k}+0.1 \mathbf{t}_{k}+\sum_{j=1}^{q} \beta_{j} \mathbf{G}_{j}+\mathbf{v}_{k},
$$

where $\mathbf{X}_{1 k}, \mathbf{X}_{2 k}, \mathbf{t}_{k}$, and $\mathbf{v}_{k}$ were set up the same way as for the Type I error evaluation. $\mathbf{G}_{1}, \mathbf{G}_{2}, \ldots, \mathbf{G}_{q}$ are the genotypes of the causal SNPs. We assumed that $30 \%$ of all variants were disease susceptibility variants, and each $\beta_{j}$ was set to $c\left|\log _{10} M A F_{j}\right|$, where $c=0.4$ [19]. We also considered a situation in which one third of the causal variants were protective (i.e., $20 \%$ disease variants and $10 \%$ protective variants), where we set $\beta_{j}=-c\left|\log _{10} M A F_{j}\right|$ for the protective variants. Again, the phenotypes for all families were simulated in the same manner, and these 1,000 sets of phenotypes for each of the 100-genotype datasets were used to evaluate the statistical power. We compared LF-KM-m5 with LF-KM-m3, LF-KM-missing, avg-F-KM, and last-F-KM.
Table 1. Type I error rates of the L-KM-m5, L-KM-m3, L-KMmissing, avg-KM, and last-KM from the population-based simulation at significance levels of $0.05,0.01,0.005$, and 0.001

\begin{tabular}{lllll}
\hline & $\alpha=0.05$ & $\alpha=0.01$ & $\alpha=0.005$ & $\alpha=0.001$ \\
\hline L-KM-m5 & 0.05004 & 0.00988 & 0.00466 & 0.00081 \\
L-KM-m3 & 0.05040 & 0.00975 & 0.00481 & 0.00091 \\
L-KM-missing & 0.05041 & 0.00954 & 0.00477 & 0.00093 \\
avg-KM & 0.04935 & 0.00941 & 0.00472 & 0.00090 \\
last-KM & 0.04789 & 0.00976 & 0.00496 & 0.00098 \\
\hline
\end{tabular}

Table 2. Type I error rates of the LF-KM-m5, LF-KM-m3, LF-KMmissing, favg-F-KM, last-F-KM), and L-KM-m5 from the familybased simulation at significance levels of $0.05,0.01,0.005$, and 0.001

$$
\alpha=0.05 \quad a=0.01 \quad \alpha=0.005 \quad \alpha=0.001
$$

\section{0 trios}

$\begin{array}{lllll}\text { LF-KM-m5 } & 0.04736 & 0.00923 & 0.00465 & 0.00071 \\ \text { LF-KM-m3 } & 0.04776 & 0.00893 & 0.00455 & 0.00073 \\ \text { LF-KM-missing } & 0.04852 & 0.00904 & 0.00461 & 0.00084 \\ \text { avg-F-KM } & 0.04870 & 0.00922 & 0.00439 & 0.00083 \\ \text { last-F-KM } & 0.04795 & 0.00932 & 0.00437 & 0.00091 \\ \text { L-KM-m5 } & 0.10023 & 0.02528 & 0.01417 & 0.00329\end{array}$

\section{0 three-generation families}

0.04903

LF-KM-m3 $\quad 0.04914$

LF-KM-missing $\quad 0.04918$

avg-F-KM $\quad 0.04958$

last-F-KM $\quad 0.04946$

$\begin{array}{lll}0.00980 & 0.00484 & 0.00099 \\ 0.00971 & 0.00480 & 0.00094 \\ 0.00990 & 0.00512 & 0.00100 \\ 0.00978 & 0.00472 & 0.00089 \\ 0.00963 & 0.00487 & 0.00080 \\ 0.04928 & 0.02885 & 0.00874\end{array}$

$\begin{array}{lllll}\text { L-KM-m5 } & 0.16505 & 0.04928 & 0.02885 & 0.00874\end{array}$

\section{GAW18 Data}

To demonstrate the utility of our proposed method for real data, we analyzed data from GAW18, which consisted of whole-genome sequence data in a pedigree-based sample with longitudinal phenotypes for blood pressure and hypertension. Although 200 replicates of simulated longitudinal data are available, we only utilized the real phenotypes. The GAW18 data is drawn from T2D-GENES Project 2, and these families were obtained from two studies: the San Antonio Family Heart Study (SAFHS) [63] and the San Antonio Family Diabetes/Gallbladder Study (SAFDGS) [64]. Only odd-numbered autosomes are available. The sequence dataset contains dosage (minor allele counts); a Merlin-based procedure was employed to impute missing dosages [65]. The dosage values can assume any decimal number from 0 to 2 , instead of 0,1 , and 2 as in the additive model. Only rare variants (MAF <0.05) were used in the analysis, and weights similar to those employed by Wu et al. [19] were used. We assigned SNPs to a gene if they were located within $5 \mathrm{~kb}$ of the gene on both sides. In total, 11,096 genes were used in the analysis.

We evaluated the association of genetic variants with diastolic and systolic blood pressure (DBP and SBP, respectively), which 


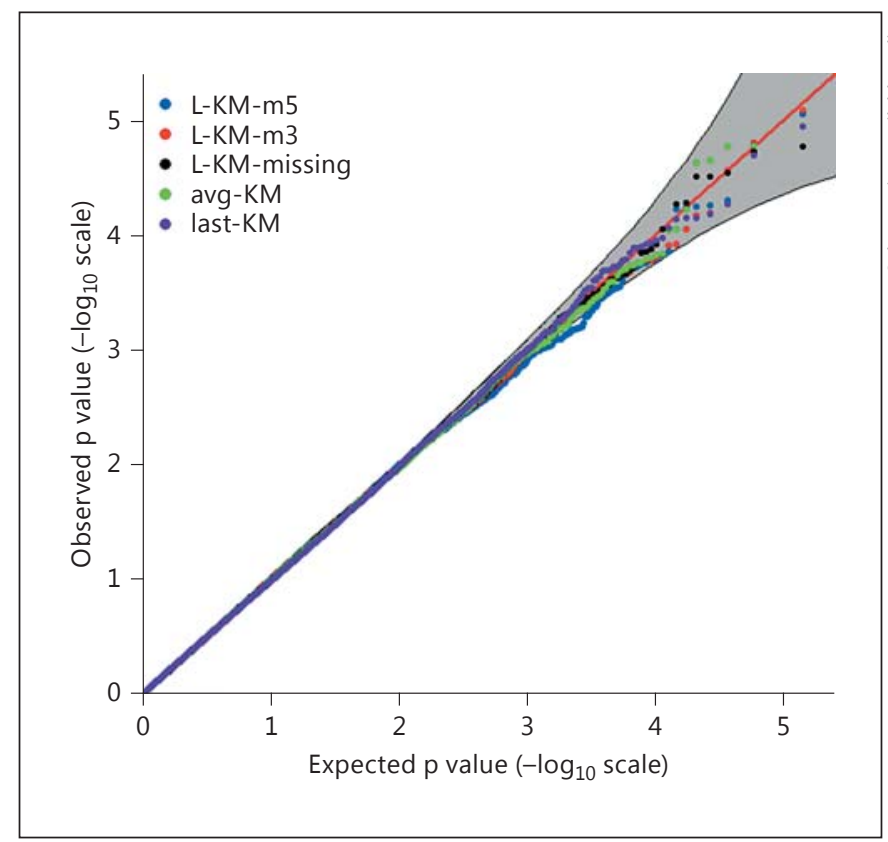

Fig. 2. QQ plot of the $\mathrm{p}$ values for population samples, with a $95 \%$ pointwise confidence band (gray area) that was computed under the assumption that the $\mathrm{p}$ values were drawn independently from a uniform $[0,1]$ distribution.

are considered heritable traits [66]. In total, 855 subjects from 20 families were used in the analysis, and each subject had up to 4 examinations (sample size at examination 1: 855, examination 2: 605, examination 3: 622, and examination 4: 233). We applied LFKM to test the association between DBP and SBP and each of the genes, with adjustment for age and sex. Some subjects had less than four observations, but this linear mixed model-based approach was flexible enough to accommodate missing observations. Under the assumption of missing at random, the incomplete data can be directly analyzed by linear mixed model [67]. Even though subjects may have missing observations at different time points, the covariance matrix for each subject is constructed from the available data at the observed time points. The linear mixed model only needs to be fitted once under the null hypothesis for one set of genome-wide sequencing data. The covariate estimates $\hat{\boldsymbol{\beta}}$, and variance-covariance matrix $\hat{\boldsymbol{\Sigma}}$ can then be saved and reused for constructing LF-KM statistics with the genotypes of each gene; therefore, the overall computation time is still fast even for genome-wide data.

\section{Results}

\section{Simulation of the Type I Error Rate}

When applied to population samples, all of the methods used (i.e., L-KM-m5, L-KM-m3, L-KM-missing, avg$\mathrm{KM}$, and last-KM) had empirical Type I error rates close to the nominal level (table 1; fig. 2). When the L-KM-m5

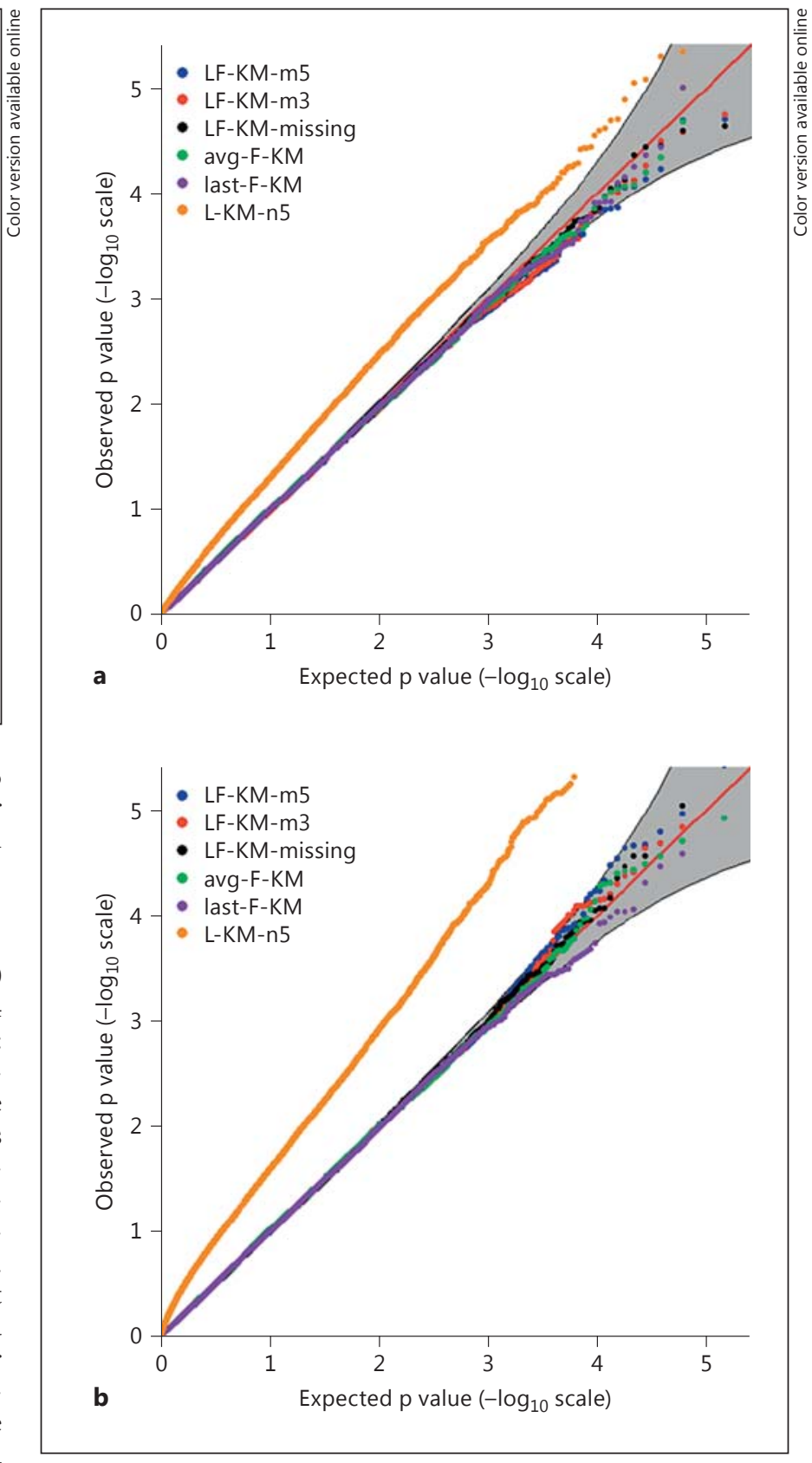

Fig. 3. QQ plots of the p values for family samples, with a $95 \%$ pointwise confidence band (gray area) that was computed under the assumption that the $\mathrm{p}$ values were drawn independently from a uniform $[0,1]$ distribution. a Trio families. b Three-generation families.

statistic was applied to family data, the Type I error rate was inflated (table 2). In contrast, LF-KM-m5, LF-KMm3, LF-KM-missing, avg-F-KM, and last-F-KM retained the desired Type I error rates. Similar patterns can be observed in the QQ plots shown in figure 3, indicating that 


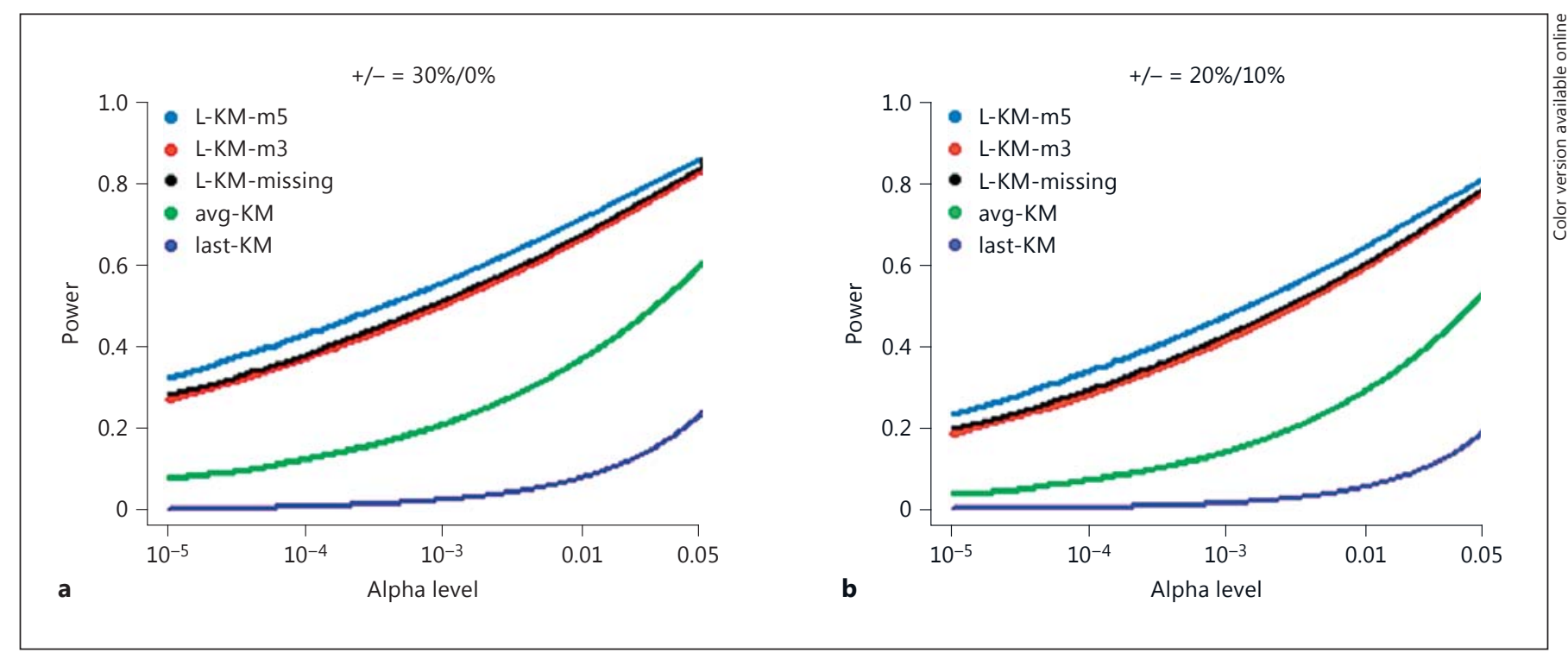

Fig. 4. Power comparison for the population samples (the a level axis uses a $\log _{10}$ scale). a A $30 \%$ disease variants scenario. b $20 \%$ disease variants and $10 \%$ protective variants scenario.

Table 3. Genes potentially associated with DBP and SBP at a significance level of $\alpha=1 \times 10^{-4}$

\begin{tabular}{|c|c|c|c|c|c|}
\hline \multicolumn{6}{|l|}{ LF-KM } \\
\hline Gene & Chr & Start & Stop & SNPs, n & $\mathrm{p}$ value \\
\hline \multicolumn{6}{|l|}{ Trait: DBP } \\
\hline DNAH9 & 17 & 11496748 & 11878485 & 1,569 & $1.49 \times 10^{-6}$ \\
\hline CDRT4 & 17 & 15334332 & 15471875 & 600 & $1.15 \times 10^{-5}$ \\
\hline FAM18B2 & 17 & 15336258 & 15471945 & 585 & $1.16 \times 10^{-5}$ \\
\hline CDRT1 & 17 & 15463798 & 15528018 & 219 & $1.51 \times 10^{-5}$ \\
\hline SCO1 & 17 & 10578654 & 10605885 & 179 & $1.79 \times 10^{-5}$ \\
\hline HS3ST3B1 & 17 & 14199400 & 14257721 & 294 & $3.21 \times 10^{-5}$ \\
\hline TRIM16 & 17 & 15526274 & 15592613 & 322 & $3.35 \times 10^{-5}$ \\
\hline CCL18 & 17 & 34386640 & 34404392 & 87 & $3.72 \times 10^{-5}$ \\
\hline INO80 & 15 & 41266078 & 41413444 & 518 & $6.44 \times 10^{-5}$ \\
\hline C17orf48 & 17 & 10595931 & 10619550 & 135 & $7.75 \times 10^{-5}$ \\
\hline FBLIM1 & 1 & 16078102 & 16118089 & 175 & $9.99 \times 10^{-5}$ \\
\hline \multicolumn{6}{|l|}{ Trait: SBP } \\
\hline PRDM10 & 11 & 129764607 & 129877730 & 416 & $4.22 \times 10^{-5}$ \\
\hline INO80 & 15 & 41266078 & 41413444 & 518 & $6.08 \times 10^{-5}$ \\
\hline TRIP10 & 19 & 6734691 & 6756537 & 75 & $7.98 \times 10^{-5}$ \\
\hline SMN1 & 5 & 70215768 & 70357324 & 63 & $8.84 \times 10^{-5}$ \\
\hline
\end{tabular}

LF-KM-m5, LF-KM-m3, LF-KM-missing, avg-F-KM, and last-F-KM control Type I error well, whereas L-KM$\mathrm{m} 5$ 's Type I error rate is more severely inflated in the data with three-generation families than that with the trio families.

\section{Statistical Power Comparison}

When we compared the power of the statistics on the population samples (fig. 4), the power of L-KM-m5 was consistently higher than that of the other methods. This was expected because L-KM-m5 made full use of the data; 


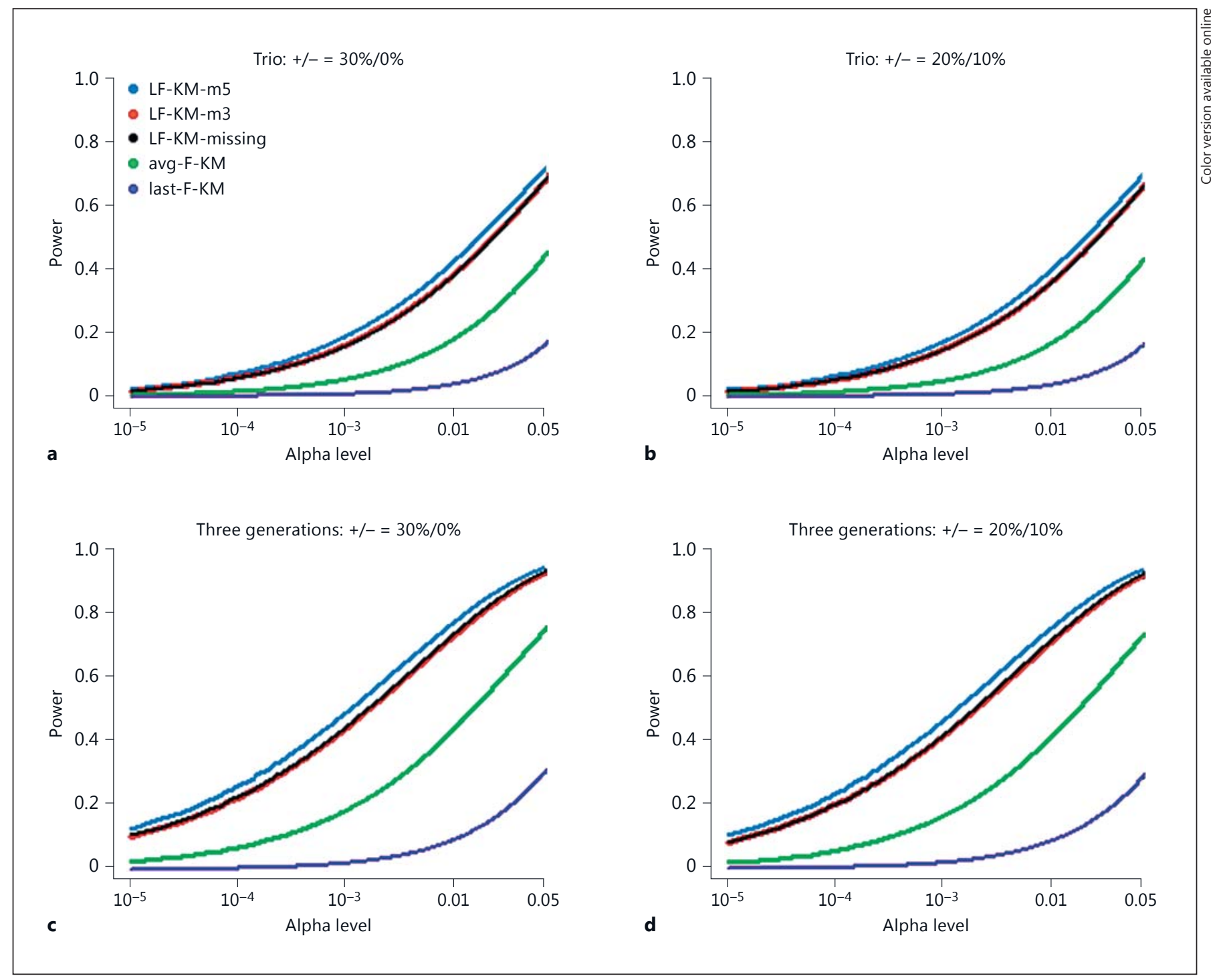

Fig. 5. Power comparison for the family samples (the a level axis uses a $\log _{10}$ scale). a A $30 \%$ disease variants scenario for trio families. b $20 \%$ disease variants and $10 \%$ protective variants scenario for trio families. c A $30 \%$ disease variants scenario for three-generation families. d $20 \%$ disease variants and $10 \%$ protective variants scenario for three-generation families.

in contrast, L-KM-m3, L-KM-missing and last-KM used only a subset of the time points, and avg-KM used the averaged phenotype, thus losing information from the longitudinal observations. Similarly, when evaluated using the family data, LF-KM-m5 outperformed the other methods as expected (fig. 5). Note that L-KM-m5 is not included in figure 5 because of its inflated Type I error rate with family data.
Table 4. Genes significant at $\alpha=0.05$ that were also shown to be associated with DBP and SBP in a large-scale GWAS [57]

\begin{tabular}{llllll}
\hline LF-KM & & & & & \\
\hline Gene & Chr & Start & Stop & SNPs, $\mathrm{n}$ & $\mathrm{p}$ value \\
\hline $\begin{array}{c}\text { Trait: DBP } \\
\quad \text { MOV10 }\end{array}$ & 1 & 113210763 & 113248368 & 124 & 0.022 \\
$\begin{array}{c}\text { Trait: SBP } \\
\text { MOV10 }\end{array}$ & 1 & 113210763 & 113248368 & 124 & 0.013 \\
SLC4A7 & 3 & 27409214 & 27530911 & 497 & 0.017 \\
\hline
\end{tabular}




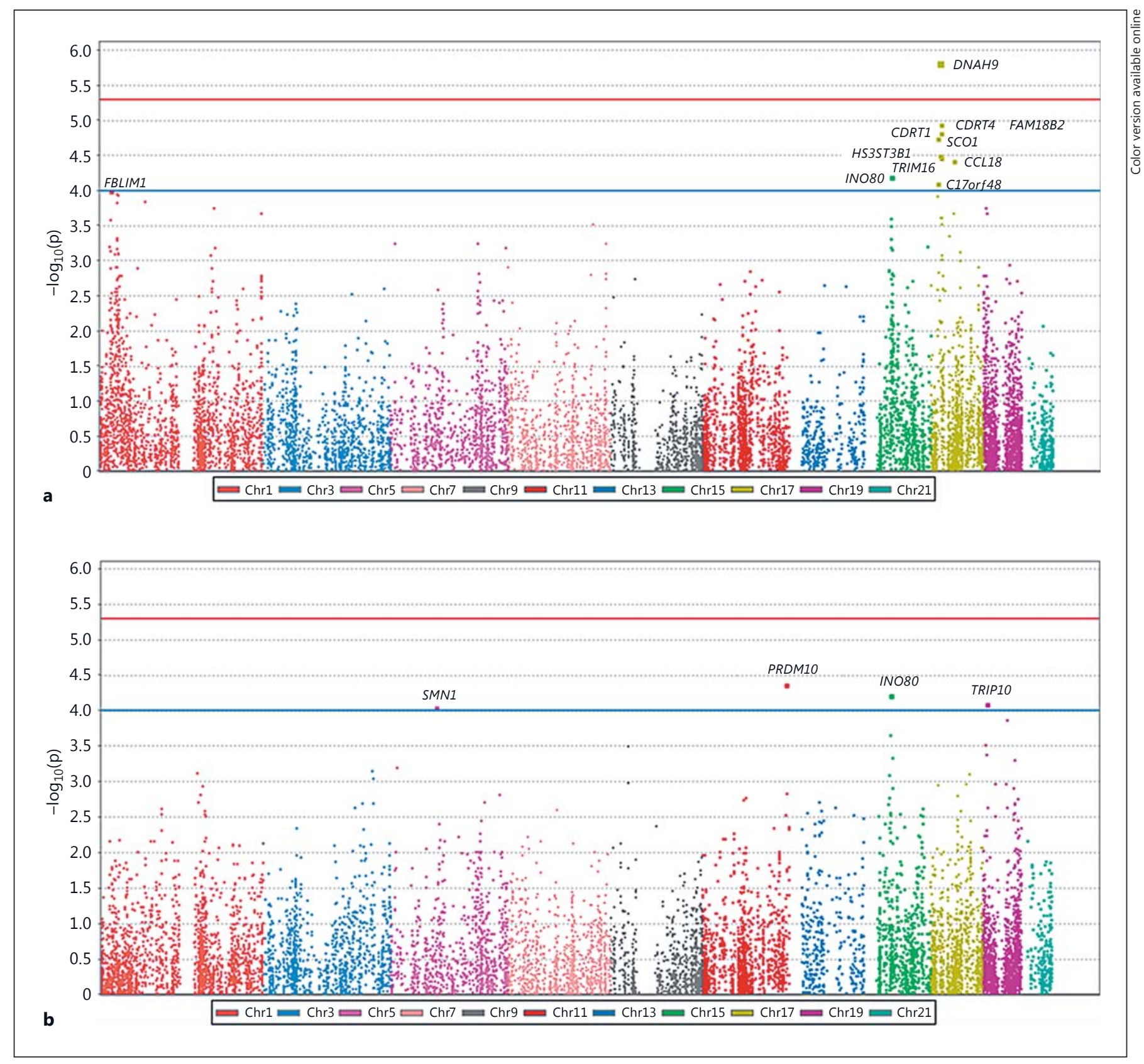

Fig. 6. $\mathbf{a}-\log _{10}$ ( $\mathrm{p}$ values) of the association between 11,096 genes and DBP. $\mathbf{b}-\log _{10}$ ( $\mathrm{p}$ values) of the association between 11,096 genes and SBP. The blue line is the suggestive significance level, $1 \times 10^{-4}$, and the red line is the stringent Bonferroni-corrected significance level, $4.5 \times 10^{-6}$. Colors refer to the online version only.

\section{GAW18 Data Analysis Results}

We used the LF-KM statistic to analyze the GAW18 data for an association between longitudinal DBP and SBP and 11,096 genes; only rare variants $(\mathrm{MAF}<0.05)$ were used. We found 11 genes that were associated with $\mathrm{DBP}$ and 4 genes that were associated with SBP, with $\mathrm{p}$ values $<0.0001$ (fig. 6 ; table 3 ). The DNAH9 gene was significantly associated with $\mathrm{DBP}$, with a $\mathrm{p}$ value $<4.5 \times 10^{-6}$ (equivalent to an a level of 0.05 after Bonferroni correction). DNAH9 contains 69 exons extending over $373 \mathrm{~kb}$, and is a rather large gene [68]. Among the significantly associated genes, INO80 was suggestively associated with 
both DBP $\left(\mathrm{p}\right.$ value $\left.=6.44 \times 10^{-5}\right)$ and SBP $(\mathrm{p}$ value $=6.08$ $\left.\times 10^{-5}\right)$. Among the genes that were previously shown to be associated with DBP and SBP in a large-scale GWAS [66], only two of them had $p$ values $<0.05$ in the LF-KM portion of our analysis (table 4 ). Note that, in our analysis, common variants were not evaluated (and only genes on odd autosomes were evaluated). This could be the reason that we did not replicate many genes that were found to be significant in the previous GWAS [66]. We also examined whether any signals were detectable when only using non-synonymous rare SNPs, and found that a region containing DNAH9, CDRT4, and FAM18B2 on chromosome 17 was associated with DBP either analyzing all rare SNPs or only non-synonymous rare SNPs (fig. 6; online suppl. fig. 1S; for all online suppl. material, see www. karger.com/doi/10.1159/000445057).

Analysis of the GAW18 data took $32.8 \mathrm{~h}$ on a single computing node with a 3-GHz CPU and 4-GB memory. Using a computer cluster with multiple nodes, we anticipate that genome-wide data analysis should be finished within hours using our proposed method.

\section{Discussion}

In this work, we developed two statistics (L-KM and LFKM) using a linear mixed model framework, which can be employed to analyze longitudinal data with quantitative traits while properly adjusting for any family structure. As set-based analysis methods which test a set of genetic variants jointly, L-KM and LF-KM share the advantages of setbased methods, such as improved power and reduced multiple testing. Another approach [24] is also able to test the association between longitudinal phenotypes and genes. In this algorithm, the test is based on collapsing markers within a gene via an aggregated index; however, this could introduce substantial noise to the summarized value. In addition, permutation is used to evaluate $p$ values, which is computationally intensive, particularly on a genome-wide scale. On the other hand, our proposed methods do not collapse markers into a single value; thus, they allow each marker to have different directions and magnitudes of effects. Our proposed kernel regression-based methods can analytically compute $\mathrm{p}$ values without resampling, leading to a substantial reduction in computation time. Furthermore, our proposed methods are computationally efficient because they are basically score tests, and thus the null model (which does not include genotypes) only needs to be fitted once for the whole genome. Different methods have higher power in different scenarios. When most of the genetic variants analyzed are causal and the direction of their effects are the same, the optimal sequence kernel association tests (SKAT-O) and burden tests have higher power [69] than SKAT. When most of the variants in a region are not causal and the directions of effects of causal variants are different, SKAT has higher power [69] than SKAT-O and burden tests. The L-KM and LF-KM statistics can be extended to the 'optimal' framework by combining with burden test statistic (see online suppl. materials for details), which is similar to the extension of SKAT to SKAT-O [69]. The optimal methods have empirical Type I error rates close to the nominal level (online suppl. fig. 3S, 4S) and increased power when casual variants are in the same direction (online suppl. fig. 5S, 6S).

In the population-based simulation studies, we showed that L-KM preserves the desired Type I error rates. When multiple measurements were available at different time points, we showed that L-KM achieves higher power than the competing approaches by using measurements at all time points. In the family-based simulation studies, we showed that using L-KM on data with related samples results in an inflated Type I error rate, while LF-KM had the correct Type I error rate because it considered the familial structure in the model. Analogously, LF-KM achieves the best power performance when using all observations. Based on our simulation study, L-KM is a good choice for genetic analysis of longitudinal data for population samples, and LF-KM is a good choice for family samples. It should be noted that LF-KM is more general and includes $\mathrm{L}-\mathrm{KM}$ as a special case, where each individual can be treated as being from one family.

The L-KM and LF-KM computation times depend on the complexity of the model which is influenced by factors such as sample size, the number of genetic variants, and the number of repeated measures. In fact, the computation time required to fit the model under the null hypothesis may not be crucial when performing a genome-wide analysis. Because L-KM and LF-KM are score tests, the estimates of fixed-effects coefficients and the covariance matrices under the null hypothesis are not related to the genetic variants. The linear mixed model under the null hypothesis only needs to be fitted once for the whole genome. The covariate estimates $\hat{\boldsymbol{\beta}}$, and variance-covariance matrix $\hat{\Sigma}$ can then be saved and reused for constructing test statistics for all the genes. On the other hand, plugging the genes one by one into the formula takes the majority of the computation time. If the number of markers in a gene is large, matrix inversion is still computationally intensive. If the total number of observations is large, the runtime may be infeasible using single computing node (online suppl. 
fig. 2S). Although parallel and powerful computing facilities are available, it is always advantageous to use fast algorithms, such as EMMA/EMMAX [58, 70], TASSEL [70], and several new fast linear mixed model algorithms [7174], to make our approach faster and more efficient.

In the real data analysis, we only included rare variants to illustrate our proposed method; we did not perform a thorough analysis and an attempt to draw biological conclusions. Therefore, we did not intend to arrive at any biological conclusions from this illustrative analysis. In practice, both common and rare variants are usually available and we must analyze both. There are different analysis strategies to handle this. One strategy is to include both common and rare variants in the set-based analysis [75]. Another strategy is to group rare variants into sets $[7-9,19,76]$ and analyze common variants individually. A third strategy is to group rare variants and common variants into different sets and test them either together or separately $[12,77-80]$. The comparison of these strategies is beyond the scope of this work and also depends on the data being analyzed.

Although the method we propose includes some assumptions, the framework is general and flexible. Covariates can be easily incorporated into the model. The L-KM and LF-KM algorithms were implemented in R (http:// www.r-project.org) and the source code is available online (http://www.pitt.edu/ qiy17/Softwares.html).

\section{Appendix}

Derivation of the KM Test in a Linear Mixed Model

Framework

For the linear mixed model, the log likelihood is

$$
l=C-\frac{1}{2} \log \left|\sum\right|-\frac{1}{2}(\mathbf{y}-\mathbf{X} \boldsymbol{\beta})^{\prime} \sum^{-1}(\mathbf{y}-\mathbf{X} \boldsymbol{\beta}) \text {. }
$$

To derive the score test for $H_{0}: \tau=0$, we take the first derivative with respect to $\tau$

$$
\begin{aligned}
\frac{d l}{d \tau}= & -\frac{1}{2} \operatorname{tr}\left(\sum^{-1} \mathbf{G W G}^{\prime}\right) \\
& +\frac{1}{2}(\mathbf{y}-\mathbf{X} \boldsymbol{\beta})^{\prime} \sum^{-1} \mathbf{G W G}^{\prime} \sum^{-1}(\mathbf{y}-\mathbf{X} \boldsymbol{\beta}),
\end{aligned}
$$

where

$$
\frac{d l}{d \tau}
$$

is the score function of $\tau$ under $H_{0}: \tau=0$. If $\sum$ is replaced with its estimate, the first term in the score function is fixed and does not depend on phenotype $\mathbf{y}$. Following the same rationale as used in the derivation of the SKAT score statistic [46-48], we used twice the second term as our test statistic.
Under the null hypothesis, the linear mixed model is $\mathbf{y}=\mathbf{X} \boldsymbol{\beta}+$ $\mathbf{u}+\boldsymbol{\varepsilon}$, and the estimates are

$$
\begin{aligned}
& \hat{\boldsymbol{\Sigma}}=\hat{\mathbf{K}}+\hat{\sigma}_{E}^{2} \mathbf{I} \\
& \hat{\boldsymbol{\beta}}=\left(\mathbf{X}^{\prime} \hat{\Sigma}^{-1} \mathbf{X}\right)^{-1} \mathbf{X}^{\prime} \hat{\Sigma}^{-1} \mathbf{y} .
\end{aligned}
$$

Replacing the variance components with their maximum likelihood estimators (MLEs), we have

$$
\mathrm{Q}=(\mathbf{y}-\mathbf{X} \hat{\boldsymbol{\beta}})^{\prime} \hat{\Sigma}^{-1} \mathbf{G W G}^{\prime} \hat{\Sigma}^{-1}(\mathbf{y}-\mathbf{X} \hat{\boldsymbol{\beta}})
$$

as the test statistic. Under the null hypothesis, the variance of the residual is

$$
\begin{aligned}
& \operatorname{Var}(\mathbf{y}-\mathbf{X} \hat{\boldsymbol{\beta}})=\operatorname{Var}\left(\mathbf{y}-\mathbf{X}\left(\mathbf{X}^{\prime} \hat{\Sigma}^{-1} \mathbf{X}\right)^{-1} \mathbf{X}^{\prime} \hat{\Sigma}^{-1} \mathbf{y}\right) \\
& =\hat{\Sigma}-\mathbf{X}\left(\mathbf{X}^{\prime} \hat{\Sigma}^{-1} \mathbf{X}\right)^{-1} \mathbf{X}^{\prime}=\mathbf{P}_{\mathbf{0}} .
\end{aligned}
$$

The statistic $\mathrm{Q}$ is a quadratic form of $\mathbf{y}-\mathbf{X} \hat{\boldsymbol{\beta}}$ and follows a mixture of $\chi^{2}$ distributions under $H_{0}$. Thus,

$$
\mathrm{Q} \sim \Sigma_{i=1}^{q} \lambda_{i} \chi_{1, i}^{2},
$$

where $\lambda_{i}$ are the eigenvalues of the matrix

$$
\mathbf{W}^{\frac{1}{2}} \mathbf{G}^{\prime} \hat{\sum}^{-1} \mathbf{P}_{\mathbf{0}} \widehat{\sum}^{-1} \mathbf{G W}^{\frac{1}{2}}
$$

[38], which can be proved by the Theorem of the Distribution of Quadratic Forms and the Theorem of Equal Eigenvalues. The $p$ values can be calculated by different algorithms, such as Davies' method [52].

The Theorem of Equal Eigenvalues states that for matrices A and $\mathrm{B}$, the eigenvalues of $\mathrm{AB}$ and $\mathrm{BA}$ are equal if one of $\mathrm{A}$ and $\mathrm{B}$ is invertible. In our case,

$$
\begin{aligned}
(\mathbf{y}-\mathbf{X} \hat{\boldsymbol{\beta}}) \sim N\left(0, \mathbf{P}_{\mathbf{0}}\right) & \text { and } \mathbf{K}_{\mathbf{0}}=\widehat{\bar{\sum}}{ }^{-1} \mathbf{G W G}^{\prime} \widehat{\mathbf{\sum}^{-1}} \\
= & \left(\mathbf{W}^{\frac{1}{2}} \mathbf{G}^{\prime} \widehat{\sum^{-1}}\right),\left(\mathbf{W}^{\frac{1}{2}} \mathbf{G}^{\prime} \widehat{\bar{\sum}}-1\right)=\mathbf{K}^{\prime} \mathbf{K} .
\end{aligned}
$$

Therefore, $\lambda_{i}$ in $\mathrm{Q} \sim \sum_{i=1}^{n} \lambda_{i} \chi_{1, i}^{2}$ are the eigenvalues of the matrix $\mathrm{K}^{\prime} \mathrm{KP}_{0}$ according to the theorem in Yuan and Bentler [49], which is equivalent to the nonzero eigenvalues $\lambda_{i}$ in $\mathrm{Q} \sim \sum_{i=1}^{q} \lambda_{i} \chi_{1, i}^{2}$ of the matrix

$$
\mathrm{KP}_{0} \mathbf{K}^{\prime}=\mathbf{W}^{\frac{1}{2}} \mathbf{G}^{\prime} \hat{\sum}^{-1} \mathbf{P}_{0} \hat{\sum}^{-1} \mathbf{G W}^{\frac{1}{2}}
$$

according to the Theorem of Equal Eigenvalues. The reason for using this matrix form is that sample size $n$ is usually larger than the number of markers $q$ in one gene, and thus the size of $\mathbf{K P}_{\mathbf{0}} \mathbf{K}^{\prime}$ is smaller than $\mathbf{K}^{\prime} \mathbf{K} \mathbf{P}_{\mathbf{0}}$. Therefore, less computation is involved when using $\mathbf{K P}_{\mathbf{0}} \mathbf{K}^{\prime}$. If sample size $n$ is smaller than the number of markers $q$, there is no need to use $\mathbf{K P}_{\mathbf{0}} \mathbf{K}^{\prime}$.

\section{Acknowledgments}

This work was supported in part by grant EPS1158862 (Q.Y., H.K.T.) and 1462990 (X.L., N.L.) from the National Science Foundation; grants GM081488, R03DE024198, R01HL092173, P60AR064172, UL1TR001417 (N.L.), 5R01GM069430-08 (N.Y.), 5R01DA025095 (X.-Y.L.), GM073766 (G.G.), R01 HG007358 (W.C.), and R01 EY024226 (W.C.) from the National Institutes of Health; grant from the Children's Hospital of Pittsburgh of the UPMC Health System; grants 102-2628-B-002-039-MY3 from the 
Ministry of Science and Technology of Taiwan, and NTU-CESRP104R7622-8 from National Taiwan University (W-Y.L.). GAW18 was provided by the T2D-GENES Consortium, which was supported by NIH grants U01 DK085524, U01 DK085584, U01 DK085501, U01 DK085526, and U01 DK085545. The content is solely the responsibility of the authors and does not necessarily represent the official views of the sponsors.

\section{Disclosure Statement}

The authors declare that they have no conflicts of interest.

\section{References}

1 Wellcome Trust Case Control Consortium: Genome-wide association study of 14,000 cases of seven common diseases and 3,000 shared controls. Nature 2007;447:661-678.

$\checkmark 2$ Hunter DJ, Kraft P, Jacobs KB, Cox DG, Yeager M, Hankinson SE, Wacholder S, Wang Z, Welch R, Hutchinson A, Wang J, Yu K, Chatterjee N, Orr N, Willett WC, Colditz GA, Ziegler RG, Berg CD, Buys SS, McCarty CA, Feigelson HS, Calle EE, Thun MJ, Hayes RB, Tucker M, Gerhard DS, Fraumeni JF Jr, Hoover RN, Thomas G, Chanock SJ: A genome-wide association study identifies alleles in FGFR2 associated with risk of sporadic postmenopausal breast cancer. Nat Genet 2007;39: 870-874.

3 Yeager M, Orr N, Hayes RB, et al: Genomewide association study of prostate cancer identifies a second risk locus at $8 \mathrm{q} 24$. Nat Genet 2007;39:645-649.

4 Hindorff LA, Sethupathy P, Junkins HA, Ramos EM, Mehta JP, Collins FS, Manolio TA: Potential etiologic and functional implications of genome-wide association loci for human diseases and traits. Proc Natl Acad Sci USA 2009;106:9362-9367.

5 Manolio TA, Brooks LD, Collins FS: A HapMap harvest of insights into the genetics of common disease. J Clin Invest 2008;118:15901605.

6 Schork NJ, Murray SS, Frazer KA, Topol EJ: Common vs. rare allele hypotheses for complex diseases. Curr Opin Genet Dev 2009; 19: 212-219.

7 Li B, Leal SM: Methods for detecting associations with rare variants for common diseases: Application to analysis of sequence data. Am J Hum Genet 2008;83:311-321.

8 Madsen BE, Browning SR: A groupwise association test for rare mutations using a weighted sum statistic. PLoS Genet 2009;5:e1000384.

9 Morgenthaler S, Thilly WG: A strategy to discover genes that carry multi-allelic or monoallelic risk for common diseases: a cohort allelic sums test (cast). Mutat Res 2007;615:28-56.

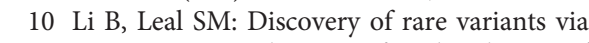
sequencing: Implications for the design of complex trait association studies. PLoS Genet 2009;5:e1000481.

-11 Price AL, Kryukov GV, de Bakker PI, Purcell SM, Staples J, Wei LJ, Sunyaev SR: Pooled association tests for rare variants in exon-resequencing studies. Am J Hum Genet 2010;86: 832-838.
12 Han F, Pan W: A data-adaptive sum test for disease association with multiple common or rare variants. Hum Hered 2010;70:42-54.

13 Morris AP, Zeggini E: An evaluation of statisti$\mathrm{cal}$ approaches to rare variant analysis in genetic association studies. Genet Epidemiol 2010;34:188-193.

14 Lin WY, Yi N, Zhi D, Zhang K, Gao G, Tiwari HK, Liu N: Haplotype-based methods for detecting uncommon causal variants with common SNPs. Genet Epidemiol 2012;36:572-582.

15 Lin WY, Yi N, Lou XY, Zhi D, Zhang K, Gao G, Tiwari HK, Liu N: Haplotype kernel association test as a powerful method to identify chromosomal regions harboring uncommon causal variants. Genet Epidemiol 2013;37:560570.

16 Lin WY, Lou XY, Gao G, Liu N: Rare variant association testing by adaptive combination of p-values. PLoS One 2014;9:e85728.

17 Yan Q, Tiwari HK, Yi N, Lin WY, Gao G, Lou XY, Cui X, Liu N: Kernel-machine testing coupled with a rank-truncation method for genetic pathway analysis. Genet Epidemiol 2014;38: 447-456.

18 Wu MC, Kraft P, Epstein MP, Taylor DM, Chanock SJ, Hunter DJ, Lin X: Powerful SNPset analysis for case-control genome-wide association studies. Am J Hum Genet 2010;86: 929-942.

19 Wu MC, Lee S, Cai T, Li Y, Boehnke M, Lin X: Rare-variant association testing for sequencing data with the sequence kernel association test. Am J Hum Genet 2011;89:82-93.

20 Aulchenko YS, Ripatti S, Lindqvist I, et al; ENGAGE Consortium: Loci influencing lipid levels and coronary heart disease risk in $16 \mathrm{Eu}$ ropean population cohorts. Nat Genet 2009; 41:47-55.

21 Kamatani Y, Matsuda K, Okada Y, Kubo M, Hosono N, Daigo Y, Nakamura Y, Kamatani $\mathrm{N}$ : Genome-wide association study of hematological and biochemical traits in a Japanese population. Nat Genet 2010;42:210-215.

22 Kathiresan S, Manning AK, Demissie S, D'Agostino RB, Surti A, Guiducci C, Gianniny L, Burtt NP, Melander O, Orho-Melander M, Arnett DK, Peloso GM, Ordovas JM, Cupples LA: A genome-wide association study for blood lipid phenotypes in the framingham heart study. BMC Med Genet 2007;8(suppl 1): S17.

23 Sabatti C, Service SK, Hartikainen AL, Pouta A, Ripatti S, Brodsky J, Jones CG, Zaitlen NA,
Varilo T, Kaakinen M, Sovio U, Ruokonen A, Laitinen J, Jakkula E, Coin L, Hoggart C, Collins A, Turunen H, Gabriel S, Elliot P, McCarthy MI, Daly MJ, Jarvelin MR, Freimer NB, Peltonen L: Genome-wide association analysis of metabolic traits in a birth cohort from a founder population. Nat Genet 2009;41:35-46.

24 Wang S, Fang S, Sha Q, Zhang S: Detecting association of rare and common variants by testing an optimally weighted combination of variants with longitudinal data. BMC Proc 2014;8:S91.

25 Furlotte NA, Eskin E, Eyheramendy S: Genome-wide association mapping with longitudinal data. Genet Epidemiol 2012;36:463-471.

26 Melton PE, Almasy LA: Bivariate association analysis of longitudinal phenotypes in families. BMC Proc 2014;8:S90.

27 Fan R, Zhang Y, Albert PS, Liu A, Wang Y, Xiong M: Longitudinal association analysis of quantitative traits. Genet Epidemiol 2012;36: 856-869.

28 Falk CT, Rubinstein P: Haplotype relative risks: an easy reliable way to construct a proper control sample for risk calculations. Ann Hum Genet 1987;51:227-233.

29 Ott J: Statistical properties of the haplotype relative risk. Genet Epidemiol 1989;6:127-130.

30 Spielman RS, McGinnis RE, Ewens WJ: Transmission test for linkage disequilibrium: the insulin gene region and insulin-dependent diabetes mellitus (IDDM). Am J Hum Genet 1993; 52:506-516.

31 Terwilliger JD, Ott J: A haplotype-based 'haplotype relative risk' approach to detecting allelic associations. Hum Hered 1992;42:337346.

32 Ott J, Kamatani Y, Lathrop M: Family-based designs for genome-wide association studies. Nat Rev Genet 2011;12:465-474.

33 George VT, Elston RC: Testing the association between polymorphic markers and quantitative traits in pedigrees. Genet Epidemiol 1987; 4:193-201.

34 Chen T, Santawisook P, Wu Z: A multi-level model for analyzing whole genome sequencing family data with longitudinal traits. BMC Proc 2014;8:S86.

$35 \mathrm{Wu}$ YY, Briollais L: Mixed-effects models for joint modeling of sequence data in longitudinal studies. BMC Proc 2014;8:S92.

-36 Zhou H, Zhou J, Sobel EM, Lange K: Fast genome-wide pedigree quantitative trait loci analysis using Mendel. BMC Proc 2014;8:S93. 
-37 Rabinowitz D, Laird N: A unified approach to adjusting association tests for population admixture with arbitrary pedigree structure and arbitrary missing marker information. Hum Hered 2000;50:211-223.

38 Chen H, Meigs JB, Dupuis J: Sequence kernel association test for quantitative traits in family samples. Genet Epidemiol 2013;37:196-204.

-39 Schifano ED, Epstein MP, Bielak LF, Jhun MA, Kardia SL, Peyser PA, Lin X: SNP set association analysis for familial data. Genet Epidemiol 2012;36:797-810.

40 Oualkacha K, Dastani Z, Li R, Cingolani PE, Spector TD, Hammond CJ, Richards JB, Ciampi A, Greenwood CM: Adjusted sequence kernel association test for rare variants controlling for cryptic and family relatedness. Genet Epidemiol 2013;37:366-376.

-41 Yan Q, Tiwari HK, Yi N, Gao G, Zhang K, Lin WY, Lou XY, Cui X, Liu N: A sequence kernel association test for dichotomous traits in family samples under a generalized linear mixed model. Hum Hered 2015;79:60-68.

-42 Yan Q, Weeks DE, Celedon JC, Tiwari HK, Li B, Wang X, Lin WY, Lou XY, Gao G, Chen W, Liu N: Associating multivariate quantitative phenotypes with genetic variants in family samples with a novel kernel machine regression method. Genetics 2015;201:1329-1339.

43 Paterson AD: Drinking from the Holy Grail: Analysis of whole-genome sequencing from the genetic analysis workshop 18. Genet Epidemiol 2014;38(suppl 1):S1-S4.

44 Chen H, Malzahn D, Balliu B, Li C, Bailey JN: Testing genetic association with rare and common variants in family data. Genet Epidemio 2014;38(suppl 1):S37-S43.

45 Cordell HJ: Summary of results and discussions from the gene-based tests group at genetic analysis workshop 18. Genet Epidemio 2014;38(suppl 1):S44-S48.

46 Kwee LC, Liu D, Lin X, Ghosh D, Epstein MP: A powerful and flexible multilocus association test for quantitative traits. Am J Hum Genet 2008;82:386-397.

47 Liu D, Lin X, Ghosh D: Semiparametric regression of multidimensional genetic pathway data: least-squares kernel machines and linear mixed models. Biometrics 2007;63:1079-1088.

$\checkmark 48$ Zhang D, Lin X: Hypothesis testing in semiparametric additive mixed models. Biostatistics 2003;4:57-74.

49 Yuan KH, Bentler PM: Two simple approximations to the distributions of quadratic forms. Br J Math Stat Psychol 2010;63:273291.

-50 Schifano ED, Epstein MP, Bielak LF, Jhun MA, Kardia SL, Peyser PA, Lin X: SNP set association analysis for familial data. Genet Epidemiol 2012;36:797-810.

51 Satterthwaite FE: An approximate distribution of estimates of variance components. Biometrics 1946;2:110-114.

52 Davies R: The distribution of a linear combination of chi-square random variables. J R Stat Soc Ser C Appl Stat 1980;29:323-333.
Kuonen D: Saddlepoint approximations for distributions of quadratic forms in normal variables. Biometrika 1999;86:929-935.

54 Pinheiro J, Bates D, DebRoy S, Sarkar D; RCore Team: nlme: linear and nonlinear mixed effects models. R package version 31-118, 2014. https://cran.r-project.org/web/packages/nlme/ index.html.

55 Vazquez AI, Bates DM, Rosa GJ, Gianola D, Weigel KA: Technical note: an R package for fitting generalized linear mixed models in animal breeding. J Anim Sci 2010;88:497-504.

56 Schaffner SF, Foo C, Gabriel S, Reich D, Daly MJ, Altshuler D: Calibrating a coalescent simulation of human genome sequence variation. Genome Res 2005;15:1576-1583.

57 Balding DJ, Nichols RA: A method for quantifying differentiation between populations at multi-allelic loci and its implications for investigating identity and paternity. Genetica 1995; 96:3-12.

58 Kang HM, Sul JH, Service SK, Zaitlen NA, Kong SY, Freimer NB, Sabatti C, Eskin E: Variance component model to account for sample structure in genome-wide association studies. Nat Genet 2010;42:348-354.

59 Lynch M, Ritland K: Estimation of pairwise relatedness with molecular markers. Genetics 1999;152:1753-1766.

60 Ritland K: Multilocus estimation of pairwise relatedness with dominant markers. Mol Ecol 2005; 14:3157-3165.

61 Yu J, Pressoir G, Briggs WH, Vroh Bi I, Yamasaki M, Doebley JF, McMullen MD, Gaut BS, Nielsen DM, Holland JB, Kresovich S, Buckler ES: A unified mixed-model method for association mapping that accounts for multiple levels of relatedness. Nat Genet 2006;38:203-208.

62 Liu N, Zhao H, Patki A, Limdi NA, Allison DB Controlling population structure in human genetic association studies with samples of unrelated individuals. Stat Interface 2011;4:317326.

63 Mitchell BD, Kammerer CM, Blangero J, Mahaney MC, Rainwater DL, Dyke B, Hixson JE, Henkel RD, Sharp RM, Comuzzie AG, VandeBerg JL, Stern MP, MacCluer JW: Genetic and environmental contributions to cardiovascular risk factors in Mexican Americans. The San Antonio Family Heart Study. Circulation 1996;94:2159-2170

-64 Hunt KJ, Lehman DM, Arya R, Fowler S, Leach RJ, Goring HH, Almasy L, Blangero J, Dyer TD, Duggirala R, Stern MP: Genome-wide linkage analyses of type 2 diabetes in Mexican Americans: The San Antonio Family Diabetes/ Gallbladder Study. Diabetes 2005;54:26552662.

65 Abecasis GR, Cherny SS, Cookson WO, Cardon LR: Merlin - rapid analysis of dense genetic maps using sparse gene flow trees. Nat Genet 2002;30:97-101
66 International Consortium for Blood Pressure Genome-Wide Association Studies; Ehret GB, Munroe PB, Rice KM, et al: Genetic variants in novel pathways influence blood pressure and cardiovascular disease risk. Nature 2011;478: 103-109.

67 Laird NM: Missing data in longitudinal studies. Stat Med 1988;7:305-315.

68 Bartoloni L, Blouin JL, Maiti AK, Sainsbury A, Rossier C, Gehrig C, She JX, Marron MP, Lander ES, Meeks M, Chung E, Armengot M, Jorissen M, Scott HS, Delozier-Blanchet CD, Gardiner RM, Antonarakis SE: Axonemal beta heavy chain dynein DNAH9: cDNA sequence, genomic structure, and investigation of its role in primary ciliary dyskinesia. Genomics 2001; 72:21-33.

69 Lee S, Wu MC, Lin X: Optimal tests for rare variant effects in sequencing association studies. Biostatistics 2012;13:762-775.

-70 Zhang Z, Ersoz E, Lai CQ, Todhunter RJ, Tiwari HK, Gore MA, Bradbury PJ, Yu J, Arnett DK, Ordovas JM, Buckler ES: Mixed linear model approach adapted for genome-wide association studies. Nat Genet 2010;42:355-360.

71 Zhou X, Stephens M: Efficient multivariate linear mixed model algorithms for genome-wide association studies. Nat Methods 2014;11:407409.

72 Lippert C, Listgarten J, Liu Y, Kadie CM, Davidson RI, Heckerman D: Fast linear mixed models for genome-wide association studies. Nat Methods 2011;8:833-835.

-73 Svishcheva GR, Axenovich TI, Belonogova NM, van Duijn CM, Aulchenko YS: Rapid variance components-based method for whole-genome association analysis. Nat Genet 2012;44:1166-1170.

74 Zhou X, Stephens M: Genome-wide efficient mixed-model analysis for association studies. Nat Genet 2012;44:821-824.

75 Ionita-Laza I, Lee S, Makarov V, Buxbaum JD, Lin X: Sequence kernel association tests for the combined effect of rare and common variants. Am J Hum Genet 2013;92:841-853.

-76 Lee S, Emond MJ, Bamshad MJ, Barnes KC Rieder MJ, Nickerson DA;NHLBI GO Exome Sequencing Project-ESP Lung Project Team; Christiani DC, Wurfel MM, Lin X: Optimal unified approach for rare-variant association testing with application to small-sample casecontrol whole-exome sequencing studies. Am J Hum Genet 2012;91:224-237.

77 Wang T, Elston RC: Improved power by use of a weighted score test for linkage disequilibrium mapping. Am J Hum Genet 2007;80:353-360.

78 Chapman J, Whittaker J: Analysis of multiple SNPs in a candidate gene or region. Genet Epidemiol 2008:32:560-566.

79 Pan W: Asymptotic tests of association with multiple SNPs in linkage disequilibrium. Genet Epidemiol 2009;33:497-507.

80 Yi N, Liu N, Zhi D, Li J: Hierarchical generalized linear models for multiple groups of rare and common variants: jointly estimating group and individual-variant effects. PLoS Genet 2011;7:e1002382. 